\title{
AHORRO ENERGÉTICO EN RIEGO POR ASPERSIÓN. PRUEBAS DE NUEVOS ASPERSORES EN BANCO AUTOMÁTICO CON VIENTO
}

\author{
Salvatierra Bellido, B. (1) (P), Morillo Velarde, R. (2), Pérez Urrestarazu, L. (3), Andrades \\ Gonzalez, J.A(4), Bermejo Corrales, J.L. (4) \\ ${ }^{1}$ Tecnólogo titular, IFAPA Centro Chipiona, (Cádiz), benito.salvatierra@juntadeandalucia.es \\ ${ }^{2}$ Investigador y Director de AIMCRA, Valladolid, r.morillo@aimcra.es \\ ${ }^{3}$ Investigador y Profesor Titular de la Universidad de Sevilla. Iperez@us.es \\ ${ }^{3}$ Técnico de AIMCRA, IFAPA Centro Chipiona, (Cádiz), lord noru@hotmail.com \\ ${ }^{4}$ Director técnico de la Zona Sur de AIMCRA, Sevilla,j.l.bermejo@aimcra.es
}

\section{Resumen}

El consumo energético en regadío es directamente proporcional a los valores objetivos de presión de una red colectiva de riego presurizada. El sistema de riego en parcela que más condiciona esta presión objetivo es el riego por aspersión. En este trabajo se ha analizado el comportamiento de los aspersores más utilizados en España en condiciones normales de funcionamiento frente a condiciones de presiones más bajas. Para ello se utilizó un banco de ensayo automático con viento y otro en condiciones confinadas sin viento. Se analizaron los aspersores en múltiples condiciones de trabajo, priorizando en el estudio la variable presión. Para ello se contempló un rango de presiones de 1,5 bar a 3.5 bar y con dos regímenes de viento: sin viento y con viento moderado. Como resultados del estudio se ofrece un análisis global de los datos obtenidos, que justifican la posibilidad de reducir la presión en riego por aspersión Además, se profundiza en resultados concretos para la reducción de la presión en el marco de riego de doce por quince metros.

\section{1-Introducción y objetivos}

En el año 2015 el Instituto de Investigación Agraria y Pesquera de Andalucía (IFAPA) y la Asociación de Investigación para la Mejora del Cultivo de la Remolacha Azucarera (AIMCRA) hicieron un convenio de colaboración para ensayar nuevas combinaciones de aspersores y boquillas en el banco de aspersores con viento del IFAPA Centro de Chipiona. El objetivo ha sido analizar distintas combinaciones de aspersores y boquillas que pudieran conseguir buenas uniformidades de riego a presiones más bajas, lo que supondría un gran ahorro energético para instalaciones individuales y en un futuro cercano para instalaciones de redes colectivas de riego. Esta línea de trabajo se abordó con el banco automático de ensayo de aspersores con viento existente en el centro IFAPA de Chipiona. El cultivo de la remolacha azucarera en España y en otros países mediterráneos va íntimamente unido al riego por aspersión, por lo que bajar los requerimientos de presión supondría bajar los costes energéticos del riego y por tanto, los costes de cultivo. Este esquema de innovación generaría resultados para los demás cultivos que utilizaran el riego por aspersión.

Para el estudio se han ensayado todos aquellos aspersores convencionales utilizados habitualmente en el cultivo de la remolacha azucarera. Estos aspersores son los más usados en España, debido a que este cultivo está implantado en las zonas más representativas de cultivos herbáceos extensivos. Se trata de un amplio trabajodonde se tienen en cuenta las condiciones reales de funcionamiento con viento. Se han incluido la mayoría de los fabricantes y contemplado todas las situaciones de riego posibles para analizar las respuestas en condiciones de bajas presiones. En fases posteriores y para 
ofrecer resultados concretos, se contará con la participación de empresas del sector interesadas en conocer el comportamiento de sus productos en condiciones dónde se optimice el consumo energético.

El objetivo final de esta línea es conseguir un ahorro de los costes de riego(energía y agua) manteniendo o mejorando la uniformidad del riego. Para ello se buscaron combinaciones de diseño de aspersores concretos ya conocidos, en condiciones de presión de trabajo reducida, dónde se asegurara una óptima Uniformidad de Distribución (UD) del agua de riego.

\section{2- Materiales y métodos}

Para la realización del trabajo se han utilizado dos bancos de ensayos de aspersores (Salvatierra et al, 2015): uno automático con viento, con datos de distribución de agua a través del modelo matricial; y otro sin viento, a través del modelo de curva radial. El objetivo de este segundo banco fue obtener datos básicos del comportamiento de un grupo de aspersores sin viento y ver cómo influía esta variable sobre el funcionamiento en condiciones reales.Los datos obtenidos de los ensayos con viento son resultados muy precisos al tratarse de un banco automático de recogida de datos continuos(Salvatierra, 2014) y con el cumplimento de la norma ISO 7749-2 que regula el método de ensayo en banco de aspersores rotativos.

Para la ejecución de los ensayos se fijaron tres valores de presión en los ensayos con viento. Las presiones utilizadas fueron: 3.5, 2.5, y 2 bares. Para el banco manual de aspersores sin viento: $3.5,2.5,2$, y 1.5 bares.

Para el banco de ensayos automático de aspersores con viento se obtuvieron 29.160 resultados de UD y para el banco manual de aspersores sin viento21.384 resultados de UD. En total suponen $\mathbf{5 0 . 5 4 4}$ resultados de UD del material ensayado. Este número de resultados viene en relación a las variables contempladas en tabla 1.

Tabla 1. Número de variables contempladas para los resultados obtenidos.

\begin{tabular}{|c|c|c|c|c|c|c|}
\hline & 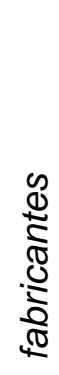 & 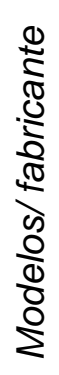 & 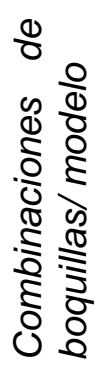 & 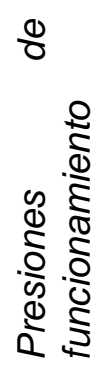 & 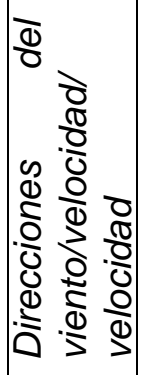 & 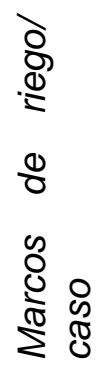 \\
\hline Banco automático de aspersores con viento & 5 & 2 & 3 & 3 & 3 & 81 \\
\hline Banco manual de aspersores sin viento & 1 & 2 & 5 & 4 & 0 & 81 \\
\hline
\end{tabular}

Los resultados que se obtiene incluyen información de variables directas medidas y otras de variables indirectas obtenidas por fórmulas empíricas y simulación. Las variables directas medidas fueron

- Fabricante aspersor.

- Modelo de aspersor.

- Boquilla principal.

- Boquilla secundaria.

- Accesorio en la boquilla.

- Caudal. 
- Presión.

- Velocidad del viento.

Y las variables indirectas fueron:

- Distancias entre aspersores.

- Distancia entre ramales.

- Disposición del marco.

- Dirección del viento.

- Índice de grosor de gota.

- Pérdidas por evaporación y arrastre.

Todos los resultados han sido clasificados para disponer de una herramienta potente de análisis y por tanto, generar resultados como los que a continuación se exponen.

\section{3- Resultados y discusión}

Un primer análisis de los resultados se focalizó sobre una muestra referida a todos los aspersores ensayados con viento en el marco de riego $12 \times 12$ (distancia entre aspersores $X$ distancia entre ramales de riego), esta muestra supone el $1,54 \%$ del total de resultados obtenidos de aspersores con viento.

En ella se obtienen los resultados siguientespara presionesmenores a 3,5 bares (que corresponde al $61 \%$ de la muestra de aspersores con viento ensayados a baja presión):

- El $48 \%$ son uniformidades aceptables y están por encima de una UD del $75 \%$

- El $33 \%$ son buenas y por encima del $80 \%$

- El $16 \%$ son excelentes y por encima del $85 \%$

Por otro lado, los ensayos a la presión nominal habitual (3,5 bares) muestran los valores siguientes: $57 \%, 36 \%$ y $12 \%$ respectivamente y según la clasificación anterior. Los valores a baja presión no son muy diferentes, y por tanto es interesante un estudio pormenorizado de los resultados generados.

A continuación se detallan los porcentajes de ensayos por cada valor de presión y marco, en relación a dos valores de uniformidad de distribución de agua críticos: UD aceptable $(>75 \%)$ y buena (>80\%). El siguiente gráfico detalla los resultados obtenidos para

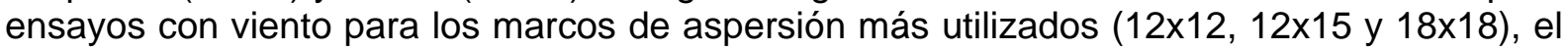
porcentaje de ensayos para cada valor de presión (3,5, 2,5 y 2 bares). 


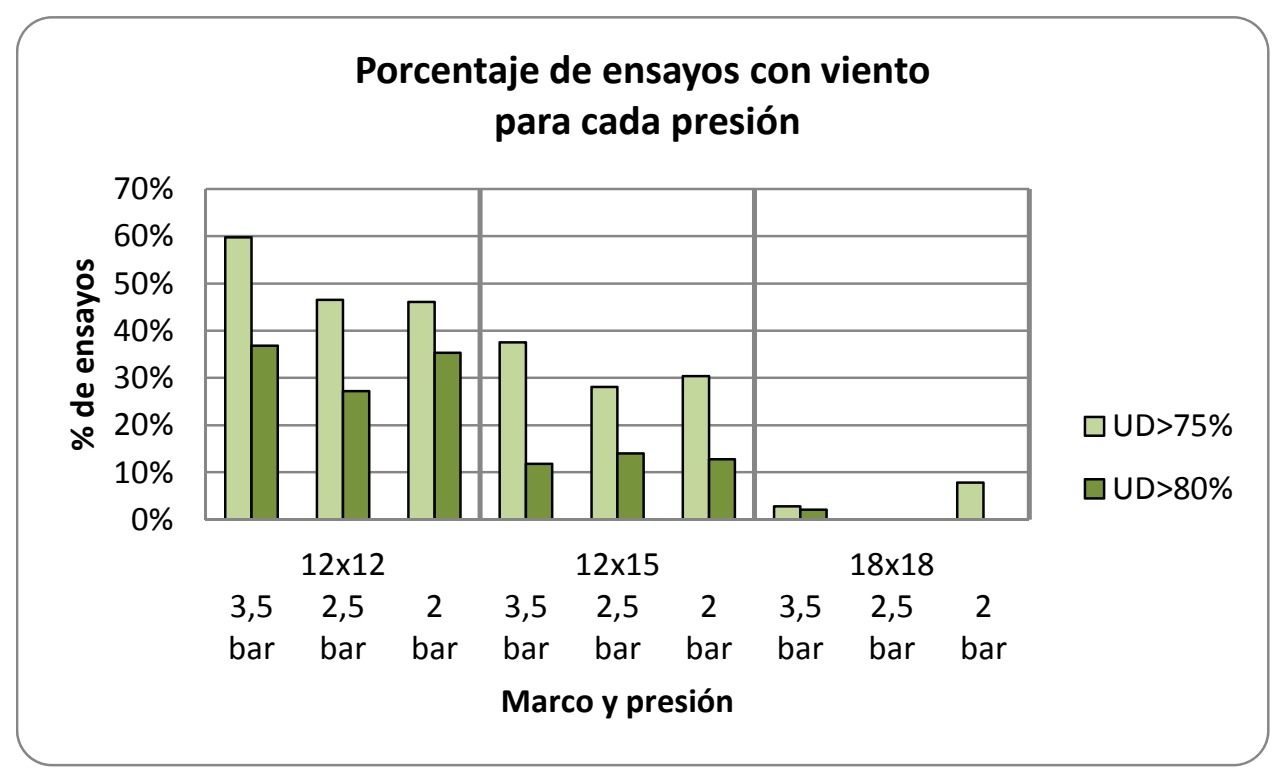

Figura 1. Porcentaje de aspersores ensayadosque tienen Uniformidad de Distribución aceptable y buena, para cada una de las presiones ensayadas y según los márcos más utilizados en riego por aspersión.

De este modo, sería posible trabajar a presiones más bajas siempre que el emisor que se utilice se haya ensayado previamente y funcione en las mismas condiciones en el ensayo.

En las gráficas siguientes, se comparará para un tipo de aspersor específico de impacto, las diferencias de funcionamiento con viento y sin viento. Éstas se refieren a los mismos criterios que la gráfica 1, pero diferenciando el porcentaje de ensayos con Uniformidad de Distribución aceptable cuando no hace viento y cuando funcionan en condiciones de viento.

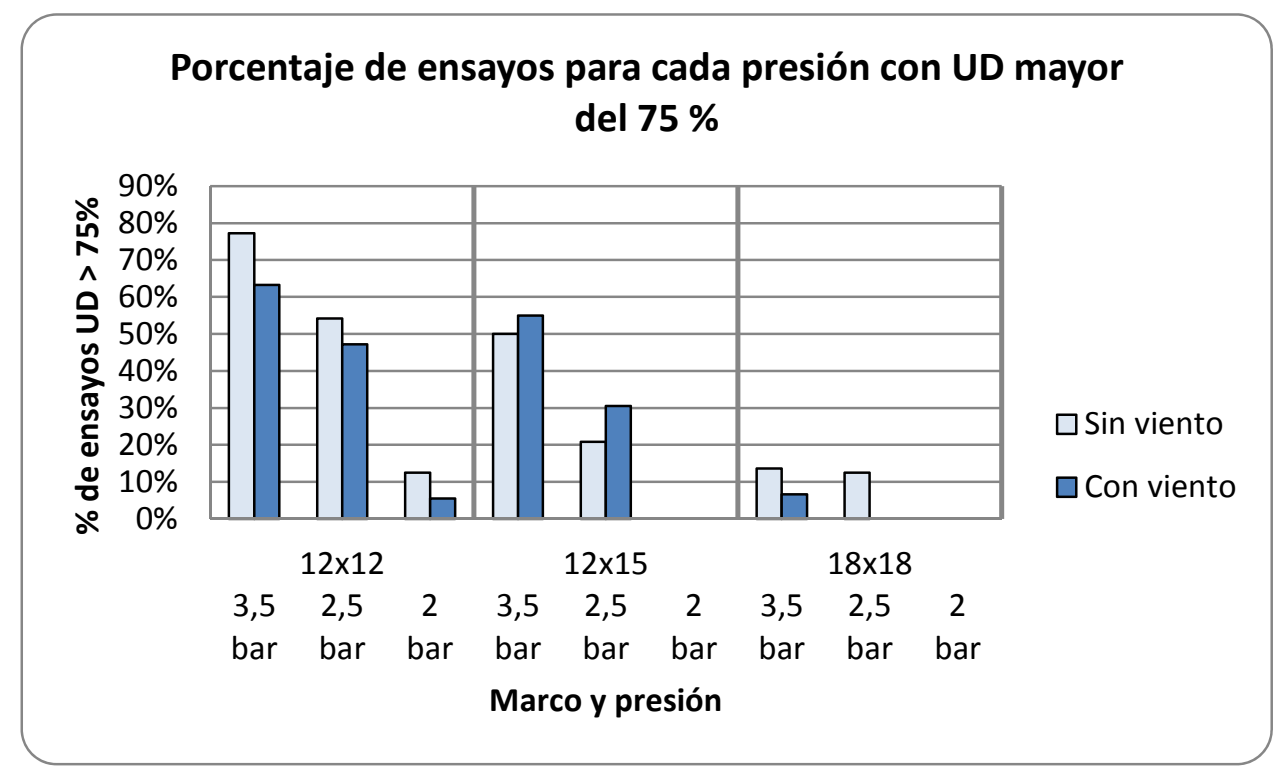

Figura 2. Ensayos realizados de un modelo convencional de aspersor de impacto para aquellos que tienen Uniformidad de Distribución aceptable, para cada una de las presiones ensayadas y según los márcos más utilizados en riego por aspersión. 
Igualmente en la siguiente gráfica se analiza el mismo tipo de aspersor pero con el criterio de obtener el porcentaje de ensayos con una Uniformidad de Distribución mayor al $80 \%$.

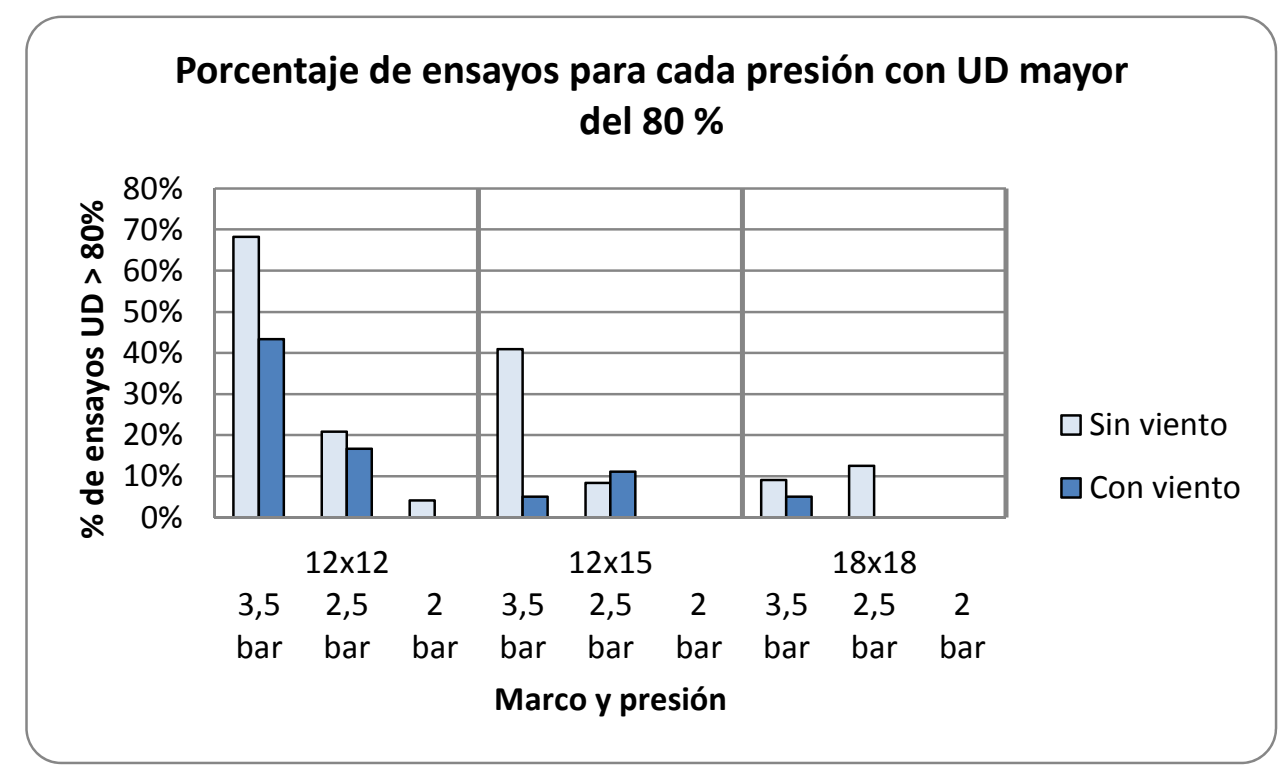

Figura 3. Ensayos realizados de un modelo convencional de aspersor de impacto para aquellos que tienen Uniformidad de Distribución buena, para cada una de las presiones ensayadas y según los márcosmás utilizados en riego por aspersión.

Según se indica en la gráfica 1 , dónde se analizan todos los ensayos con viento, se aprecian unos resultados para el marco $12 \times 15$ no tan buenos como en el marco de $12 \times$ 12pero mejores que para el marco de $18 \times 18$. Por ello, se podría bajar la presión, ya que existe en torno a $13 \%$ de los ensayos que tienen buena uniformidad tanto a 3,5 como en 2,5 y 2 bares de presión. Esta circunstancia es única para el marco $12 \times 15$ y para riego con viento moderado, entre 0,9 y $2,9 \mathrm{~m} / \mathrm{s}$.

En las siguientes tablas se muestran los aspersores y las variable con las que se han obtenido los valores de Uniformidad de Distribución. La primera tabla hace referencia a los obtenidos para una presión de funcionamiento de 3,5 bares, la segunda para 2,5 y la tercera para 2 bares.

Los ensayos señalados en verde oscuro, corresponden aaquellos aspersores,ycondiciones de funcionamientoquepresentanbuena uniformidad en las tres presiones ensayadas. Los señalados con color verde claro son aquellos que tienen buena uniformidad sólo a 3,5 y a 2,5 bares. Para no identificar la marcar comercial del aspersor, se ha decido codificarla como se puede apreciar en la columna de fabricante y modelo. 
Tabla 2. Ensayos de aspersores con buena uniformidad en marco de $12 \times 15$, a 3,5 bares con viento.

\begin{tabular}{|c|c|c|c|c|c|c|c|c|c|c|c|}
\hline S & 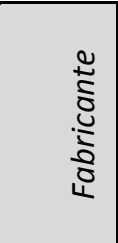 & $\begin{array}{l}\frac{0}{8} \\
\frac{8}{2} \\
\sum^{2}\end{array}$ & $\begin{array}{l}\stackrel{0}{0} \\
\text { ¿े }\end{array}$ & 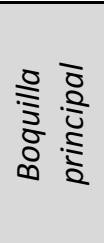 & 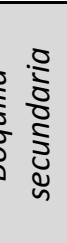 & t) & 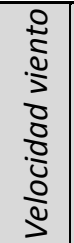 & 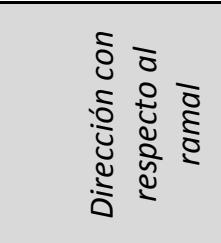 & $\begin{array}{l}\frac{1}{5} \\
\frac{5}{0} \\
\frac{0}{0} \\
0\end{array}$ & 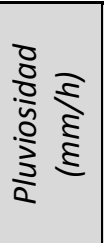 & 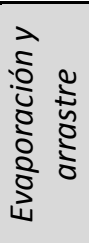 \\
\hline $81 \%$ & vVvVvV & gggg & ectangular & $11 / 64$ & & & 1,9 & Perpendicular & 361 & 7,56 & $18 \%$ \\
\hline $83 \%$ & $x x x x x x$ & bbbb & ectangular & $5 / 32$ & si & si & 2,9 & Paralelo & 527 & 8,48 & $28 \%$ \\
\hline $84 \%$ & $x x x x x x$ & bbbb & gular & $5 / 32$ & si & sí & 2,9 & Perpendicular & 527 & 8,48 & $28 \%$ \\
\hline $82 \%$ & $x x x x x x$ & bbbb & gular & 9/64 & si & si & 2,6 & Perpendicular & 350 & 7,50 & $26 \%$ \\
\hline $82 \%$ & $x x x x x x$ & bbbb & lar & $5 / 32$ & si & si & 2,9 & Paralelo & 527 & 8,48 & $28 \%$ \\
\hline $82 \%$ & $x x x x x x$ & bbbb & lar & $5 / 32$ & si & si & 2,9 & Perpendicular & 527 & 8,48 & $28 \%$ \\
\hline $82 \%$ & $x x x x x x$ & bbbb & ular & $9 / 64$ & si & si & 2,6 & Perpendicular & 350 & 7,50 & $26 \%$ \\
\hline $83 \%$ & $x x x x x x$ & $\operatorname{cccc}$ & Triangular & $5 / 32$ & si & si & 2,7 & Perpendicular & 1408 & 7,82 & $26 \%$ \\
\hline $81 \%$ & yyyyyy & aaaa & Rectangular & $9 / 64$ & & si & 1,5 & Paralelo & 909 & 5,05 & $17 \%$ \\
\hline $84 \%$ & yyyyyy & hhhh & Rectangular & $3 / 16$ & si & si & 2,0 & Perpendicular & 1927 & 10,71 & $17 \%$ \\
\hline $86 \%$ & yyyyyy & hhhh & Triar & $3 / 16$ & si & si & 2,0 & Perpendicular & 1927 & 10,71 & $17 \%$ \\
\hline $86 \%$ & zzzzzz & ffff & ngular & $5 / 32$ & si & si & 1,6 & Oblicuo & 1520 & 8,45 & $18 \%$ \\
\hline $84 \%$ & zzzzzz & $\mathrm{ffff}$ & gular & $5 / 32$ & si & si & 1,6 & Par & 1520 & 8,45 & $18 \%$ \\
\hline $90 \%$ & zzzzzz & ffff & gular & $5 / 32$ & si & si & 1,6 & Perpendic & 1520 & 8,45 & $18 \%$ \\
\hline $86 \%$ & zzzz & $\mathrm{ffff}$ & & $5 / 32$ & si & si & 1,6 & Oblicuo & 1520 & 8,45 & $18 \%$ \\
\hline $82 \%$ & zzzz & $\mathrm{ffff}$ & & $5 / 32$ & si & si & 1,6 & Paralelo & 1520 & 8,45 & $18 \%$ \\
\hline $91 \%$ & zzzzzz & $\mathrm{ffff}$ & Triangular & $5 / 32$ & si & si & 1,6 & Perpendic & 1520 & 8,45 & $18 \%$ \\
\hline
\end{tabular}

Tabla 3. Ensayos de aspersores con buena uniformidad en marco de $12 \times 15$, a 2,5 bares con viento.

\begin{tabular}{|c|c|c|c|c|c|c|c|c|c|c|c|}
\hline S & 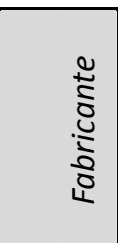 & $\begin{array}{l}\frac{0}{2} \\
\frac{0}{0} \\
\Sigma\end{array}$ & 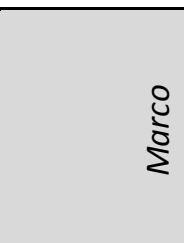 & 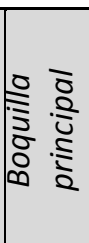 & 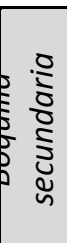 & ) & 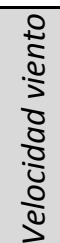 & 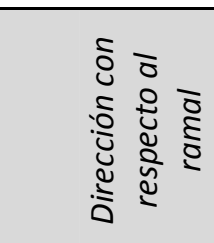 & $\begin{array}{l}\frac{5}{5} \\
\frac{0}{0} \\
0 \\
0 \\
0\end{array}$ & 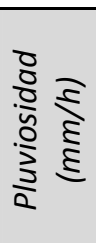 & 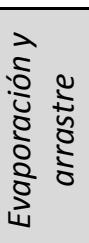 \\
\hline $84 \%$ & $x x x x x x$ & $\mathrm{bbbb}$ & Rectangular & $5 / 32$ & si & si & 2,1 & Paralela & 1.293 & 7,18 & $13 \%$ \\
\hline $86 \%$ & $x x x x x x$ & bbbb & Rectangular & $5 / 32$ & si & si & 2,1 & Perpendicular & 1.293 & 7,18 & $13 \%$ \\
\hline $84 \%$ & $x x x x x x$ & bbbb & Rectangular & $9 / 64$ & si & si & 1,5 & Oblicuo & 1.221 & 6,78 & $12 \%$ \\
\hline $87 \%$ & $x x x x x x$ & bbbb & Rectangular & $9 / 64$ & si & si & 1,5 & Paralela & 1.221 & 6,78 & $12 \%$ \\
\hline $87 \%$ & $x x x x x x$ & bbbb & Rectangular & $9 / 64$ & si & si & 1,5 & Perpendicular & 1.221 & 6,78 & $12 \%$ \\
\hline $82 \%$ & $x x x x x x$ & bbbb & Triangular & $5 / 32$ & si & si & 2,1 & Paralela & 1.293 & 7,18 & $13 \%$ \\
\hline $85 \%$ & $x x x x x x$ & bbbb & Triangular & $5 / 32$ & si & si & 2,1 & Perpendicular & 1.293 & 7,18 & $13 \%$ \\
\hline $84 \%$ & $x x x x x x$ & bbbb & Triangular & 9/64 & si & si & 1,5 & Oblicuo & 1.221 & 6,78 & $12 \%$ \\
\hline $84 \%$ & $x x x x x x$ & bbbb & Triangular & 9/64 & si & si & 1,5 & Paralela & 1.221 & 6,78 & $12 \%$ \\
\hline $89 \%$ & $x x x x x x$ & bbbb & Triangular & $9 / 64$ & si & si & 1,5 & Perpendicular & 1.221 & 6,78 & $12 \%$ \\
\hline $82 \%$ & yyyyyy & aaaa & Rectangular & $5 / 32$ & si & si & 1,0 & Oblicuo & 1.325 & 7,36 & $8 \%$ \\
\hline $84 \%$ & yyyyyy & aaaa & Triangular & $5 / 32$ & si & si & 1,0 & Oblicuo & 1.325 & 7,36 & $8 \%$ \\
\hline $83 \%$ & yyyyyy & aaaa & Triangular & $5 / 32$ & si & si & 1,0 & Paralela & 1.325 & 7,36 & $8 \%$ \\
\hline $82 \%$ & yyyyyy & aaaa & Triangular & $5 / 32$ & si & si & 1,0 & Perpendicular & 1.325 & 7,36 & $8 \%$ \\
\hline $82 \%$ & zzzzzz & ffff & Rectangular & $5 / 32$ & si & si & 2,1 & Perpendicular & 1.277 & 7,09 & $14 \%$ \\
\hline $82 \%$ & zzzzzz & $\mathrm{ffff}$ & Triangular & $5 / 32$ & si & si & 2,1 & Perpendicular & 1.277 & 7,09 & $14 \%$ \\
\hline
\end{tabular}


Tabla 4. Ensayos de aspersores con buena uniformidad en marco de $12 \times 15$, a 2 bares con viento.

\begin{tabular}{|c|c|c|c|c|c|c|c|c|c|c|c|}
\hline$\Im$ & 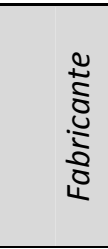 & $\begin{array}{l}\frac{0}{8} \\
\frac{0}{2} \\
\frac{0}{\Sigma}\end{array}$ & $\begin{array}{l}\text { ¿े } \\
\text { के } \\
\sum\end{array}$ & 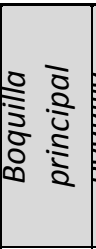 & 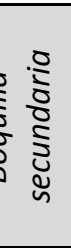 & $\frac{0}{5}$ & 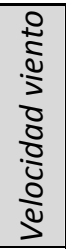 & 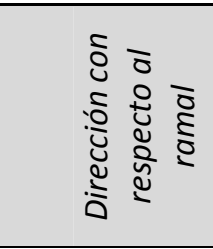 & $\begin{array}{l}\frac{1}{\vdots} \\
\frac{0}{0} \\
\frac{0}{0}\end{array}$ & $\begin{array}{l}\frac{0}{0} \\
\frac{0}{n} \\
\frac{0}{2} \\
\frac{1}{2} \\
\frac{1}{2}\end{array}$ & 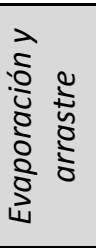 \\
\hline $84 \%$ & $x x x x x x$ & $\mathrm{bbbb}$ & Rectangular & 9/64 & si & si & 0,9 & Oblicuo & 967 & 5,37 & $6 \%$ \\
\hline $86 \%$ & $x x x x x x$ & bbbb & Rectangular & $9 / 64$ & si & si & 0,9 & Paralela & 967 & 5,37 & $6 \%$ \\
\hline $86 \%$ & $x x x x x x$ & bbbb & Rectangular & $9 / 64$ & si & si & 0,9 & Perpendicular & 967 & 5,37 & $6 \%$ \\
\hline $88 \%$ & $x x x x x x$ & $\mathrm{bbbb}$ & Triangular & $5 / 32$ & si & si & 0,8 & Oblicuo & 1.141 & 6,34 & $5 \%$ \\
\hline $88 \%$ & $\operatorname{XXXXXX}$ & $\mathrm{bbbb}$ & Triangular & $5 / 32$ & si & si & 0,8 & Paralela & 1.141 & 6,34 & $5 \%$ \\
\hline $90 \%$ & xxxxxx & $\mathrm{bbbb}$ & Triangular & $5 / 32$ & si & si & 0,8 & Perpendicular & 1.141 & 6,34 & $5 \%$ \\
\hline $89 \%$ & $x x x x x x$ & $\mathrm{bbbb}$ & Triangular & $9 / 64$ & si & si & 0,9 & Oblicuo & 967 & 5,37 & $6 \%$ \\
\hline $88 \%$ & $x x x x x x$ & $\mathrm{bbbb}$ & Triangular & 9/64 & si & si & 0,9 & Paralela & 967 & 5,37 & $6 \%$ \\
\hline $86 \%$ & $x x x x x x$ & $\mathrm{bbbb}$ & Triangular & $9 / 64$ & si & si & 0,9 & Perpendicular & 967 & 5,37 & $6 \%$ \\
\hline $82 \%$ & zzzzzz & ffff & Rectangular & $5 / 32$ & si & si & 1,6 & Oblicuo & 1.125 & 6,25 & $8 \%$ \\
\hline $88 \%$ & zzzzzzz & ffff & Rectangular & $5 / 32$ & si & si & 1,6 & Perpendicular & 1.125 & 6,25 & $8 \%$ \\
\hline $81 \%$ & zzzzzz & ffff & Triangular & $5 / 32$ & si & si & 1,6 & Oblicuo & 1.125 & 6,25 & $8 \%$ \\
\hline $87 \%$ & zzzzzzz & ffff & Triangular & $5 / 32$ & si & si & 1,6 & Perpendicular & 1.125 & 6,25 & $8 \%$ \\
\hline
\end{tabular}

Una vez comprobado en los resultados anteriores la viabilidad de bajar los niveles de presión en determinados emisores y en situaciones concretas, se muestra un análisis global comparativo del comportamiento de los aspersores ensayados en distintas presiones y para los distintos valores de viento.

El objetivo de dicho análisis es poder conocer cómo influyen las presiones en los distintos rangos de valores de velocidad de viento. Igual que en los resultados expuestos en la figura 1, se van a diferenciar los tres marcos más utilizados en el riego por aspersión. En las figuras 4, 5 y 6, se muestran las correspondencias entre los valores deUD frente a la variable viento y para cada una las presiones ensayadas.ese han ajustado curvaspolinómicas de quinto grado. La finalidad de esta gráfica es una visión global de la tendencia de la Uniformidad de Distribución para las distintas presiones. 


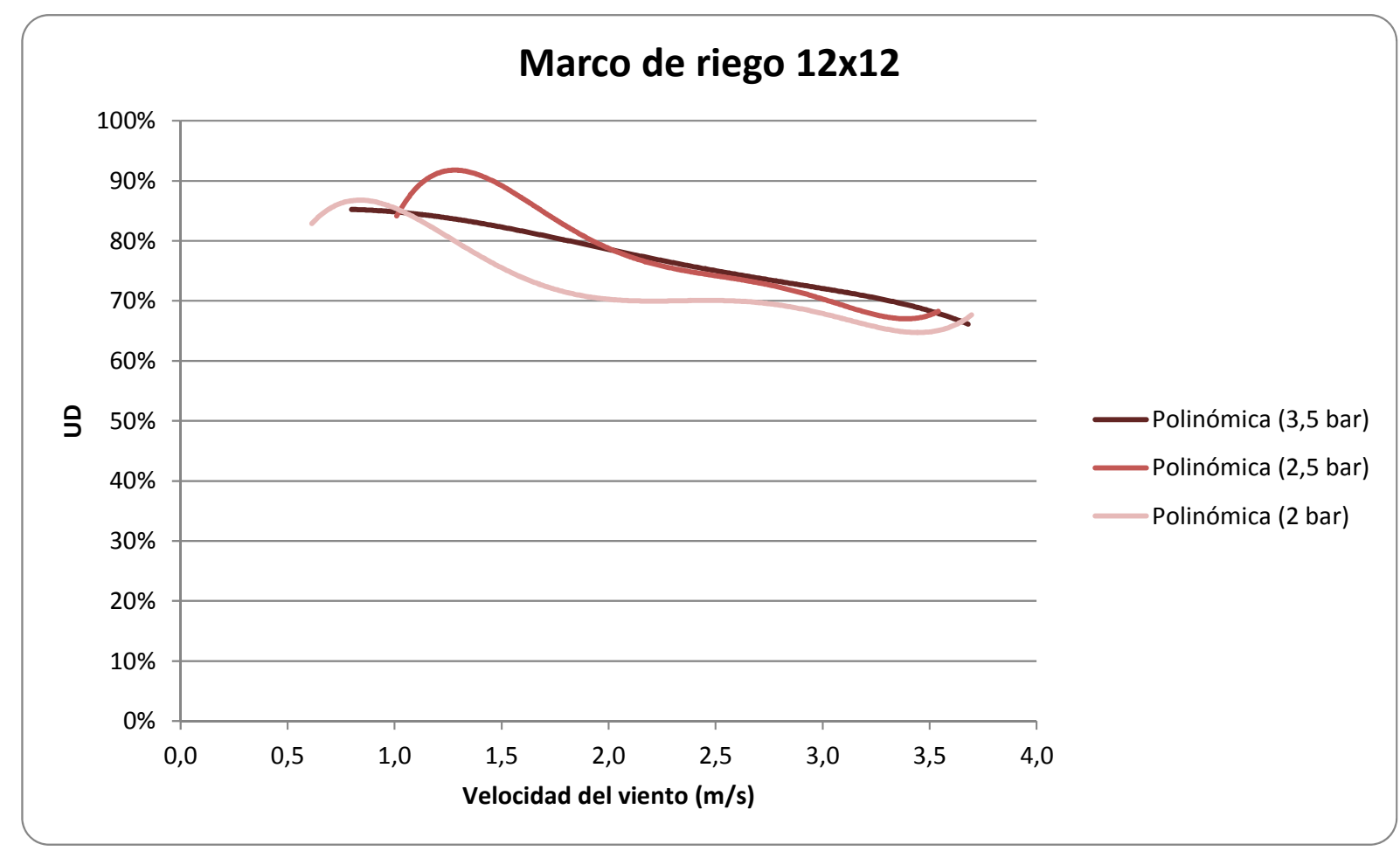

Figura 4. Gráficas de las líneas de tendencia polinómica de grado 5 resultante de los datos de UD en relación a la velocidad del viento y para los tres valores de presiones ensayadas para el marco de riego de 12×12.

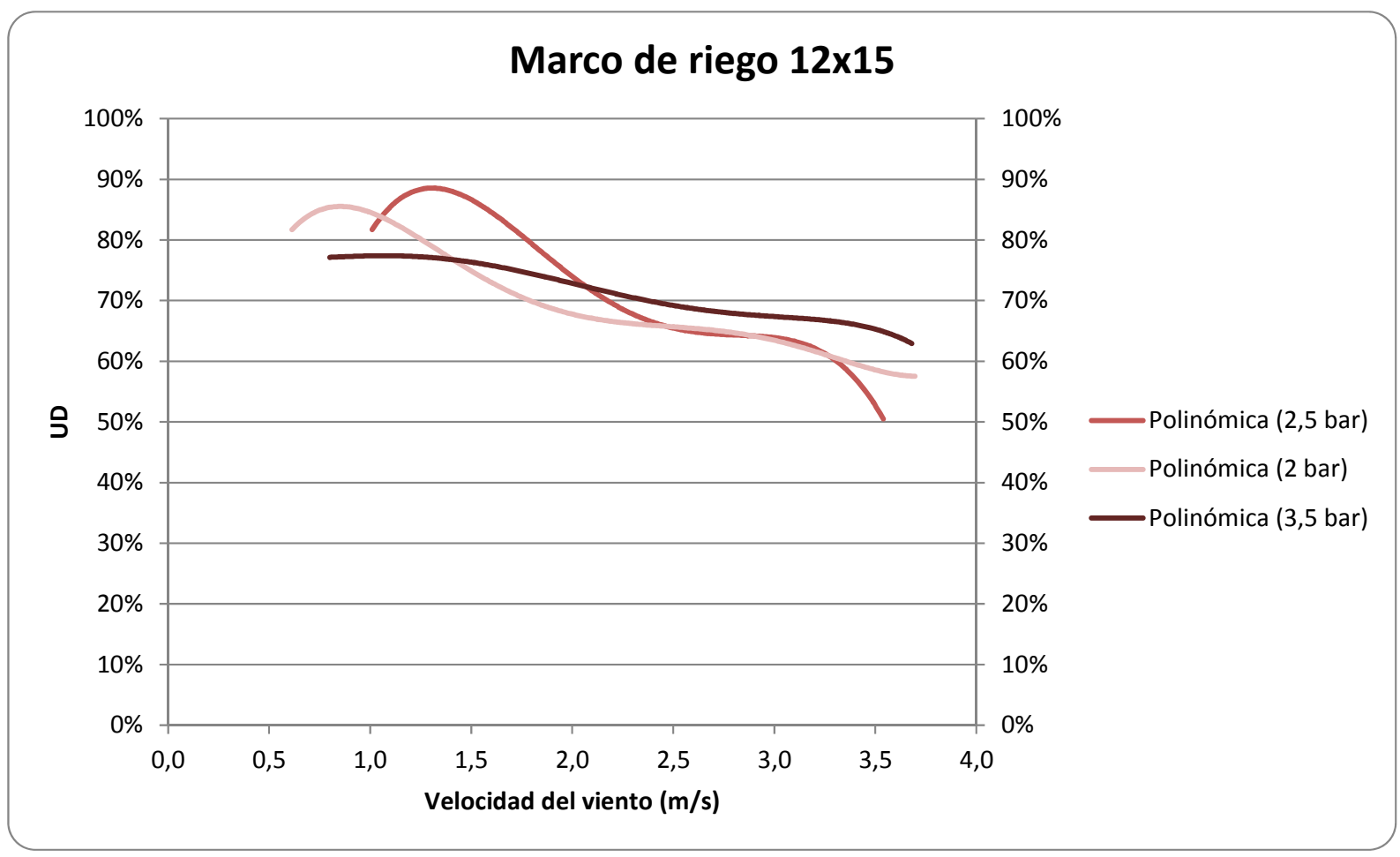

Figura 5. Gráficas de las líneas de tendencia de los datos de UD en relación a la velocidad del viento y para los tres valores de presiones ensayadas para el marco de riego de $12 \times 15$. 


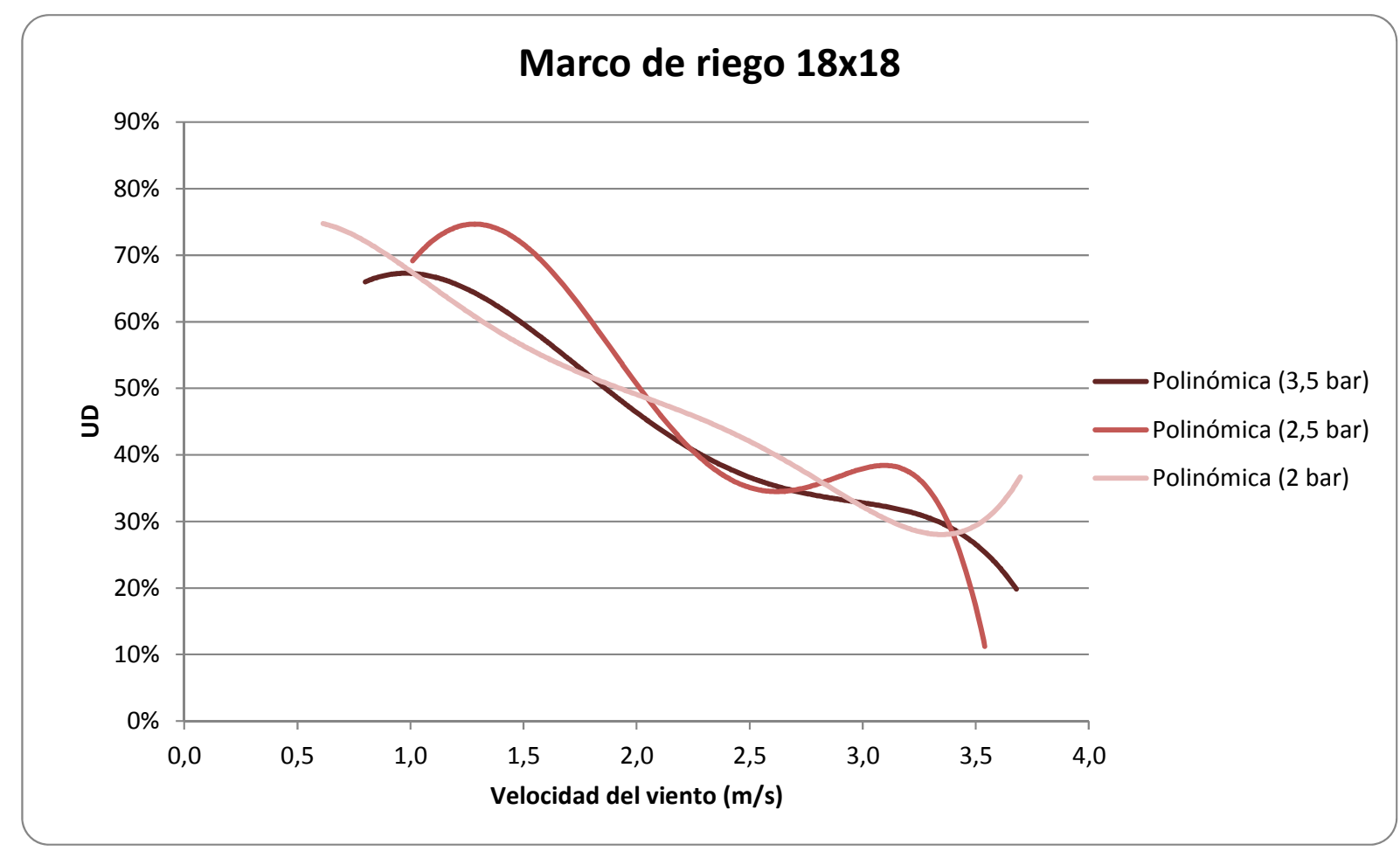

Figura 6. Gráficas de las líneas de tendencia de los datos de UD en relación a la velocidad del viento y para los tres valores de presiones ensayadas para el marco de riego de $18 \times 18$.

La gran cantidad de datos obtenidos representa una herramienta muy útil para realizar consultas concretas sobre situaciones particulares. Según los resultados presentados, para marcos más pequeños $(12 \times 12$ y $12 \times 15)$ es posible bajar las presiones hasta 2,5 bares, siempre que la velocidad de viento estuviera por debajo de $2 \mathrm{~m} / \mathrm{s}$. En los marcos mayorestambién sería posible, pero con valores de velocidad de viento más restrictivos. Esta aproximación generalista jamás debe considerarse como una recomendación para todos los casos de modo que habrá que recurrir a ensayos similares a los descritos que se correspondan con las condiciones a evaluar.

\section{4- Conclusiones y recomendaciones}

La gran cantidad de información generada representa una gran ayuda para realizarun asesoramientoal regante específico de cada caso, mejorando la uniformidad del riego y posibilitando un ahorro energético.

Vistos los resultados, existela posibilidadde bajar la presión de funcionamiento para los sistemas de riego por aspersión, siempre que se haya ensayado previamente el sistema con los condicionantes existentes en la realidad.

Para la obtención de valores aceptables o incluso buenos de Uniformidad de Distribución, intervienen en mayor o menor medida todas las variables tenidas en cuenta. Como se ha podido comprobar, en muchos casos, incluso depende del fabricante y del modelo del aspersor. 
Los datos podrían ser más numerosos para cubrir toda la casuística del mercado, por tanto, se ofrece la posibilidad a los fabricantes de realizar más ensayos y utilizarel conjunto de resultados generados para crear una aplicación que sirva para ayudar a optimizarel diseño de los riegos por aspersión.

\section{6- Bibliografía}

De Juan, J. Tarjuelo, J. Valiente, M. Garcia, P. (1994): Non-uniformity and management of sprinkler irrigation systems. Proceedings of the third congress of the European Society for Agronomy, Padova University, Abano-Padova, Italy, 18-22 September 1994.; 1994. 102-103.

ISO 7749-1:1986, Equipos de riego-Aspersores rotativos-Parte 1: Diseño y exigencias de funcionamiento.

ISO 7749-2:1990, Equipo de riego - Aspersores Rotativos - Parte 2: Uniformidad de distribución y métodos de prueba.

Merrian, J.L. Y Keller, J. (1978): Farm irrigation system evaluation: a guide for management. UTAH StateUniversity. Logan. Utah. USA.

Salvatierra, B. (2010). Uniformidad de riego en las instalaciones de riego. Boletín trimestral SAR. No 14. Enero, marzo 2010.

Salvatierra, B. (2014).Banco automático de ensayo de aspersorescon viento. XXXII Congreso Nacional de Riegos. Madrid.

Salvatierra, B. Andrades, J.A, Gómez, E. Viqueira,S. (2015). Resultados de ensayos del banco automático de aspersores.XXXIII Congreso Nacional de Riegos. Valencia.

Salvatierra, B. Andrades, J.A. (2015). Mejoras en el riego por aspersión. Revista AIMCRA, 122: $18-22$.

Sánchez, I. Faci, J. M. Zapata, N. (2011).The effects of pressure, nozzle diameter and meteorological conditions on the performance of agricultural impact sprinklers.Agricultural Water Management; 2011. 102: 1, 13-24.

Seginer, I. Nir, D. And Bernuth, R. (1991).Simulation of wind-distorded sprinkler patterns.J. Irrig. DrainEng., 117(2), 285-306.

Tarjuelo Martín Benito, J. M. (1992). El riego por aspersión: diseño y funcionamiento. Albacete: Caja de Albacete; [Toledo]. Ediciones de la Universidad de Castilla-La Mancha, D.L.

Tarjuelo Martín Benito, J. M. (2005). El riego por aspersión y su tecnología. Ediciones MundiPrensa. 581 pp.

Tarjuelo, J. M. Carrion, P. Valiente, M. (1994).Simulation of irrigation distribution by sprinkler under windy conditions.Investigacion Agraria, Produccion y Proteccion Vegetales; 9: 2, 255272.

Zapata, N. Playan, E. Martinez-Cob, A. Sanchez, I. Faci, J. M. Lecina, S. (2008).From onfarm solid-set sprinkler irrigation design to collective irrigation network design in windy areas.AgriculturalWater Management; 2007. 87: 2, 187-199. 